\title{
EFECTO DE CUATRO ESPECIES DE HONGOS MICORRIZÓGENOS ARBUSCULARES EN LA PRODUCCIÓN DE FRUTOS DE TOMATE
}

\author{
Juan Francisco Ley-Rivas ${ }^{1 / *}$, Jorge Alberto Sánchez ${ }^{*}$, Nancy Esther Ricardo ${ }^{*}$,Esther Collazo* \\ Palabras clave: Hongos micorrizógenos arbusculares; micorrizas; Glomus; tomate; índice de cosecha. \\ Keywords: Arbuscular mycorrhizal fungi; mycorrhizae; Glomus; tomato; harvest index.
}

Recibido: 04/08/14

\section{RESUMEN}

Se evaluó el efecto de los hongos micorrizógenos arbusculares (HMA) autóctonos Glomus sp. 1, Glomus sp. 2, Glomus clarum y Glomus intraradices en el cultivo de tomate (Solanum lycopersicum L. Var., amalia) en condiciones controladas, mediante las variables de crecimiento altura, diámetro del tallo, cantidad de hojas, flores y frutos, fitomasa seca aérea y subterránea y masa fresca de los frutos asimismo las variables micorrízicas que incluyen colonización, densidad visual, cuantificación de endófito, micelio externo y esporas. Las cepas Glomus sp. 1 y Glomus intraradices fueron las más eficientes, con valores del índice de cosecha de 65 y 56,3\% y masa fresca de los frutos de 166,7 y 131,8 g, respectivamente. Se destaca que la cepa Glomus sp. 1 siempre presentó valores fúngicos menores que G. intraradices.
Aceptado: 08/01/15

\begin{abstract}
Effect of four species of arbuscular mycorrhizal fungi on the production of tomato fruits. The effect of native arbuscular mycorrhizal fungi (AMF) Glomus sp. 1, Glomus sp. 2, Glomus clarum and Glomus intraradices on tomato (Solanum lycopersicum L. Var., amalia) was evaluated under controlled conditions by means of growth variables (height, stem diameter, number of leaves, flowers and fruits, dry aerial and underground biomass and fresh mass of fruits) and the mycorrhizal variables (colonization, visual density, quantification of endophyte, external mycelium and spores). The strains Glomus sp. 1 and Glomus intraradices were the most efficient, with harvest index values of 65 and $56.3 \%$ and fruit fresh weight of 166.7 and $131.8 \mathrm{~g}$, respectively. It is emphasized that the fungal strain Glomus sp. 1 always presented lower values than strain Glomus intraradices.
\end{abstract}

Autor para correspondencia. Correo electrónico: jley@ecologia.cu

* Instituto de Ecología y Sistemática, Ministerio de Ciencia, Tecnología y Medio Ambiente, Carretera de Varona 11835 e/ Oriente y Lindero, Calabazar, Boyeros, La Habana 19, C.P. 11900. La Habana, Cuba. 


\section{INTRODUCCIÓN}

Los hongos micorrizógenos arbusculares (HMA) influyen en la función y la productividad de las comunidades vegetales (Bever 2002, Irving y Cameron 2009); son los simbiontes más comunes de las raíces y su ubicación en la interfase entre raíz-suelo, los hace un grupo funcional muy importante en la biota del suelo por intervenir en los procesos de los ecosistemas (Gianinazzi et ál. 2010). Forman simbiosis mutualistas obligadas con numerosas plantas donde se ubican muchas de interés agrícola.

El micelio externo de los HMA constituye extensiones muy finas de las raíces que amplían su influencia en el suelo y crean una red que puede alcanzar hasta $30 \mathrm{~m}$ de hifas fúngicas por gramo de suelo (Wilson et ál. 2009). El efecto multiplicador de los HMA se relaciona con una mayor absorción de nutrientes, agua y fósforo (Azcóny Barea 1992, Koide y Kabir 2000), con el incremento de la tolerancia a la sequía (Smith y Read 2008, Marulanda y Barea 2009, Navarro et ál. 2010a,b), con la resistencia a la salinidad (Porras et ál. 2009) y con una mejor protección de los cultivos contra los organismos patogénicos (Baar 2008, Hao et ál. 2009).

El cultivo del tomate puede presentar una producción superior anual a 92,9 millones de toneladas lo que lo clasifica como una de las hortalizas de mayor importancia económica en el mundo. En Cuba es la hortaliza principal ya que representa el $50 \%$ del área total cultivable, se siembran anualmente 63000 ha con una producción de 802600 ton equivalentes a un rendimiento promedio de 12,74 ton.ha ${ }^{-1}$ (Ley 2012).

Por la importancia del cultivo y los efectos beneficiosos de los HMA y su empleo cada vez mayor como biofertilizantes, se analizó el efecto de 4 cepas autóctonas de HMA en la producción de biomasa y frutos a través de la evaluación de variables de crecimiento altura, diámetro del tallo, número de flores y de frutos, masa seca y fresca de la fitomasa aérea, de la raíz principal y de las raicillas así como micorrízicas colonización, densidad visual de endófito, cuantificación de los micelios interno y externo al igual que el conteo de esporas.

\section{MATERIALES Y MÉTODOS}

Cultivo seleccionado. Se seleccionó el cultivo de tomate (Solanum lycopersicum L. Var., amalia) por ser una de las hortalizas de alto consumo e interés económico para Cuba.

Selección de cepas. Se seleccionaron 4 cepas autóctonas de HMA procedentes de Sabanalamar, Pinar del Río, Glomus sp. 1 (IES 122), Glomus sp. 2 (IES 123), Glomus clarum (Nicolson \& Schenck) (IES 125) y Glomus intraradices (Shenck \& Smith) (IES 127), de la Colección Cubana de Hongos Micorrizógenos Arbusculares (CCHMA) que se encuentra en el Instituto de Ecología y Sistemática (IES) de Cuba.

Sustrato. Se utilizaron macetas de 1,3 $\mathrm{dm}^{3}$ que se llenaron con sustrato formado por una mezcla de suelo pardo con carbonato, de $\mathrm{pH}$ $\left(\mathrm{H}_{2} \mathrm{O}\right): 7,6$; MO:4,4\%, P:12 $\mu \mathrm{g} . \mathrm{g}^{-1}$, CCB:28 Cmol. $\mathrm{kg}^{-1}$ y arena sílice con partículas $<4 \mathrm{~mm}$ en proporción 2:1 v/v. Ambos componentes fueron esterilizados a vapor fluente durante $1 \mathrm{~h}$ en 3 días consecutivos con el objetivo de eliminar los microorganismos existentes.

Se preparó una solución a partir de una mezcla de suelo - agua en proporción 1:5 v/v a través de papel de filtro Whatman $\mathrm{N}^{\circ} .1$ (Orozco 2002). Todos los tratamientos incluyendo los controles sin micorrizas se inocularon con $10 \mathrm{ml}$ por maceta del filtrado del suelo del inóculo de micorrizas para reintroducir las poblaciones microbianas excepto los propágulos de micorrizas.

Diseño experimental. Los experimentos se realizaron en condiciones de casa de vegetación, con techo de cristal, paredes laterales con mallas plásticas de porosidad de $2 \mathrm{~mm}$ y se aplicó riego, hasta saturación, en días alternos, la temperatura interna varía en 25 y $38^{\circ} \mathrm{C}$.

Se desarrolló un diseño experimental completamente aleatorizado con 5 repeticiones para 
cada uno de los tratamientos que incluyeron un testigo y 4 cepas de HMA en 75 macetas con lecturas a 60, 90 y 120 días. Se sembró una semilla o planta por cada maceta, la cual se inoculó con 500 esporas de la cepa de HMA colocada directamente en los nidos de siembra excepto en los testigos. El cultivo se efectuó en el período entre octubre 2010 y enero 2011.

Variables analizadas. Se midieron las variables de crecimiento: altura, diámetro del tallo, número de flores y de frutos, fitomasa seca aérea, de la raíz principal y de las raicillas y se calculó la tasa de crecimiento relativo para la fitomasa seca total (TCRs) (Valladares 2004) y el índice de cosecha (IC) (Beable 1987), el cual se evaluó con base en los valores absoluto de la biomasa del fruto y de estructuras vegetativas con respecto a la biomasa total (biomasa subterránea, aérea y del fruto) y se calcula por la siguiente fórmula:

Índice de cosecha $=$ valores absolutos de la biomasa del fruto por planta/ valores absolutos de la biomasa total (biomasa subterránea, aérea y del fruto) $\mathrm{x} 100=\%$

Los sustratos secos de cada réplica de forma individual se mezclaron bien y se homogenizaron tomándose $100 \mathrm{~g}$ de cada uno y se procesó según la metodología de Herrera et ál.
(2004) para determinar las cantidades de micelio externo (ME), endófito (ED), esporas y los porcentajes de colonización micorrízica (CM) o frecuencia (Giovanetti y Mosse 1980) y densidad visual (DV) o intensidad de colonización una vez teñidas las raicillas por el método de Phillips y Hayman (1970).

Análisis estadístico. A los datos obtenidos se les comprobó la normalidad mediante la prueba de ajuste de Kolmogorov-Smirnov y la homogeneidad de las varianzas a través de la prueba de Bartlett. Los resultados de los tratamientos se procesaron mediante análisis de varianza (ANOVA) de clasificación simple y las diferencias entre tratamientos se determinaron por medio de la prueba de Rangos Múltiples de Duncan.

\section{RESULTADOS}

\section{Variables micorrízicas}

Colonización micorrízica: las 4 cepas mostraron altos niveles de CM superiores al 63\%. La cepa Glomus sp. 1 presentó los valores estables más bajos mientras que $G$. intraradices fue más estable con los valores más altos (Cuadro 1) donde destacan diferencias significativas con el resto de los tratamientos a los 90 y 120 días.

Cuadro 1. Efecto de la inoculación con 4 especies de HMA en el número de flores, número de tomates, masa fresca del fruto e índice de cosecha en plantas de tomate (Solanum lycopersicum L. Var., amalia) a los 60, 90 y 120 días de cultivo.

\begin{tabular}{lcccccc}
\hline & \multicolumn{3}{c}{ Colonización micorrízica (\%) } & \multicolumn{3}{c}{ Densidad visual (\%) } \\
Tratamientos & 60 & 90 & 120 & 60 & 90 & 120 \\
\hline Glomus sp 1 & $73 \mathrm{ab}$ & $69 \mathrm{c}$ & $71 \mathrm{~b}$ & 2,2 & $1,8 \mathrm{c}$ & $2,1 \mathrm{~b}$ \\
Glomus sp 2 & $71 \mathrm{~b}$ & $79 \mathrm{~b}$ & $63 \mathrm{c}$ & 2,9 & $3,7 \mathrm{~b}$ & $1,5 \mathrm{c}$ \\
G. clarum & $84 \mathrm{a}$ & $66 \mathrm{c}$ & $71 \mathrm{~b}$ & 3,2 & $2,1 \mathrm{c}$ & $2,2 \mathrm{~b}$ \\
G. intraradices & $81 \mathrm{~b}$ & $95 \mathrm{a}$ & $92 \mathrm{a}$ & 3,2 & $5,7 \mathrm{a}$ & $4,1 \mathrm{a}$ \\
\hline
\end{tabular}

Letras diferentes representan diferencias significativas entre los tratamientos para $\mathrm{p}<0,05$. 
Densidad visual: a los 60 días no existieron diferencias significativas entre los valores para todos los tratamientos. Los valores fueron similares entre sí, con excepción de la cepa $G$. intraradices la cual a los 90 y 120 días llegó a duplicarlos significativamente (Cuadro 1). La cepa Glomus sp. 1 mantuvo siempre los niveles de densidad visual más bajos. Esta variable muestra un comportamiento similar a los observados para la CM, se obtuvieron valores similares en Glomus sp. 1, Glomus sp. 2 y G. clarum.

Endófito: se observó un incremento continuo del endófito producido por las cepas Glomus sp. 1 y G. clarum. Los tratamientos con Glomus sp. 2 y G. intraradices presentan un incremento sustancial hasta los 90 días con descenso a los 120 días. En general, se observan diferencias significativas entre los tratamientos en los tiempos en que se realizaron las mediciones (Cuadro 2).

Micelio externo: se observaron diferencias significativas entre los tratamientos (Cuadro 2). La cepa G. intraradices en todos los casos fue la que produjo mayores valores, distanciándose de las otras cepas que presentan resultados más próximos. Los mayores valores se registraron a los 90 días, con excepción de la cepa Glomus sp. 2 que lo presentó a los 120 días.

Cuadro 2. Endófito arbuscular y micelio externo en plantas de tomate (Solanum lycopersicum L. Var., amalia) a los 60, 90 y 120 días de cultivo.

\begin{tabular}{lcccccc}
\hline \multicolumn{1}{r}{ Tratamientos } & \multicolumn{3}{c}{ Endófito $\left(\mathrm{mg} . \mathrm{l}^{-1}\right)$} & \multicolumn{3}{c}{ Micelio externo $\left(\mathrm{mg} . \mathrm{l}^{-1}\right)$} \\
& 60 & 90 & 120 & 60 & 90 & 120 \\
\hline Glomus sp. 1 & $2,6 \mathrm{~b}$ & $5,0 \mathrm{~b}$ & $6,2 \mathrm{~b}$ & $22,6 \mathrm{~b}$ & $25,5 \mathrm{~b}$ & $20,6 \mathrm{~b}$ \\
Glomus sp. 2 & $4,1 \mathrm{ab}$ & $13,2 \mathrm{a}$ & $7,0 \mathrm{~b}$ & $24,4 \mathrm{~b}$ & $22,4 \mathrm{~b}$ & $31,9 \mathrm{a}$ \\
G. clarum & $4,8 \mathrm{a}$ & $8,1 \mathrm{~b}$ & $13,5 \mathrm{a}$ & $22,2 \mathrm{~b}$ & $26,0 \mathrm{~b}$ & $22,1 \mathrm{~b}$ \\
G. intraradices & $5,2 \mathrm{a}$ & $15,2 \mathrm{a}$ & $14,6 \mathrm{a}$ & $39,4 \mathrm{a}$ & $48,2 \mathrm{a}$ & $34,4 \mathrm{a}$ \\
\hline
\end{tabular}

Letras diferentes representan diferencias significativas entre los tratamientos para $\mathrm{p}<0,05$.

Esporas: el conteo de esporas mostró que a los 60 días no existieron diferencias significativas entre los tratamientos; sin embargo, a los 90 y 120 días la cepa G. intraradices produjo aproximadamente de 5 a 20 veces más esporas que las otras especies, las cuales mantuvieron en todo el ciclo vegetativo de la planta una cantidad de esporas estable (Cuadro 3).

Cuadro 3. Densidad de esporas en plantas de tomate (Solanum lycopersicum L. Var., amalia) a los 60, 90 y 120 días de cultivo.

\begin{tabular}{lccc}
\hline \multicolumn{1}{c}{ Tratamientos } & \multicolumn{3}{c}{ Número de esporas (esporas.kg-1) } \\
\hline Glomus sp. 1 & $60 \mathrm{~d}$ & $90 \mathrm{~d}$ & $120 \mathrm{~d}$ \\
Glomus sp. 2 & 2280 & $1980 \mathrm{~b}$ & $1900 \mathrm{~b}$ \\
G. clarum & 1440 & $1760 \mathrm{~b}$ & $1760 \mathrm{~b}$ \\
G. intraradices & 2380 & $1200 \mathrm{~b}$ & $2520 \mathrm{~b}$ \\
\hline & 1620 & $39380 \mathrm{a}$ & $13080 \mathrm{a}$ \\
\hline
\end{tabular}

Letras diferentes representan diferencias significativas entre los tratamientos para $\mathrm{p}<0,05$. 


\section{Variables de crecimiento}

Altura: las plantas con HMA a los 60 días mostraron diferencias significativas con respecto al testigo y no entre ellas (Cuadro 4). A los $90 \mathrm{y}$ 120 días no existe diferencias significativas entre los tratamientos micorrizados, Glomus sp. 1 fue el menos efectivo para este parámetro.

Número de hojas: a los 60 días (Cuadro 4) los tratamientos micorrízicos difieren con respecto al testigo, pero a los 90 y 120 días la diferencia desaparece, observándose 2 grupos, el primero de ellos con tendencia a producir mayor biomasa aérea Glomus sp. 2 y Glomus clarum y el otro con el menor follaje en el que se encuentra el testigo, Glomus sp. 1 y G. intraradices.

Diámetro del tallo: existen diferencias significativas entre los tratamientos micorrízicos y el testigo a los 60 días (Cuadro 4). A los 90 y 120 días no existe diferencias significativas entre los tratamientos pero el valor del testigo es inferior a los tratamientos micorrizados.

Cuadro 4. Efecto de la inoculación con 4 especies de HMA, en la altura, número de hojas y diámetro del tallo en plantas de tomate (Solanum lycopersicum L. Var., amalia) a los 60, 90 y 120 días de cultivo.

\begin{tabular}{|c|c|c|c|c|c|c|c|c|c|}
\hline \multirow{2}{*}{ Tratamientos } & \multicolumn{3}{|c|}{ Altura (cm) } & \multicolumn{3}{|c|}{ Número de hojas } & \multicolumn{3}{|c|}{ Diámetro del tallo (mm) } \\
\hline & $60 d$ & $90 \mathrm{~d}$ & $120 \mathrm{~d}$ & $60 d$ & $90 d$ & $120 \mathrm{~d}$ & $60 d$ & $90 \mathrm{~d}$ & $120 d$ \\
\hline Testigo & $35,9 \mathrm{~b}$ & 77,0 & 87,4 & $8,6 \mathrm{~b}$ & $18,6 \mathrm{~b}$ & $23,4 \mathrm{~b}$ & $4,1 \mathrm{~b}$ & 5,5 & 5,6 \\
\hline Glomus sp1 & $56,8 \mathrm{a}$ & 70,9 & 73,2 & $14,8 \mathrm{a}$ & $19,2 \mathrm{ab}$ & $24,4 \mathrm{~b}$ & $5,1 \mathrm{a}$ & 5,9 & 6,7 \\
\hline Glomus sp2 & 49,9 a & 81,0 & 88,2 & $14,6 \mathrm{a}$ & $28,2 \mathrm{a}$ & $34,8 \mathrm{ab}$ & $5,6 \mathrm{a}$ & 5,8 & 6,5 \\
\hline G. clarum & 53,9 a & 82,6 & 85,0 & $15,4 \mathrm{a}$ & $25,2 \mathrm{ab}$ & $41,4 \mathrm{a}$ & 5,9 a & 6,1 & 6,1 \\
\hline \multirow[t]{2}{*}{ G. intraradices } & $61,2 \mathrm{a}$ & 78,9 & 85,9 & $17,8 \mathrm{a}$ & $20,8 \mathrm{ab}$ & $28,0 \mathrm{ab}$ & $5,7 \mathrm{a}$ & 6,3 & 7,0 \\
\hline & & NS & NS & & & & & NS & NS \\
\hline
\end{tabular}

Letras diferentes representan diferencias significativas entre los tratamientos para $\mathrm{p}<0,05$. NS (no existe diferencias significativas).

Fitomasa seca subterránea: los resultados obtenidos de la masa seca total de las raíces y las raicillas (Cuadro 5) fueron similares en su comportamiento. A los 60 días, el testigo difiere con los tratamientos micorrízicos al presentar el valor más bajo para ambas variables. En el segundo período los tratamientos con Glomus sp. 2 y G. clarum se diferencian significativamente de los otros y los valores de las plantas micorrizadas son mayores al testigo. A los 120 días el testigo y Glomus sp. 1 alcanzan los valores más bajos y las diferencias con los otros tratamientos son mayores con respecto a los períodos anteriores.
Fitomasa seca aérea: en el primer período presentó diferencias apreciables entre los tratamientos, el testigo muestra un valor muy bajo con respecto a los tratamientos micorrízicos (Cuadro 6). A los 90 días, los pesos de los tallos y las hojas en los tratamientos micorrizados fueron superiores, estadísticamente significativos a los mostrados por el testigo excepto para Glomus sp. 1 que presentó valores similares a este y no diferentes estadísticamente de Glomus sp. 2 y G. clarum.

A los 120 días los valores con la cepa Glomus sp. 1 no difieren estadísticamente del testigo y de $G$. intraradices, cuyos valores son inferiores a los de Glomus sp. 2 y G. clarum pero mayores que el testigo. 
Cuadro 5. Efecto de la inoculación, con 4 especies de HMA, en el total de las raíces (masa seca) en plantas de tomate (Solanum lycopersicum L. Var., amalia) a los 60, 90 y 120 días de cultivo.

\begin{tabular}{lccc|ccc}
\hline \multirow{2}{*}{ Tratamientos } & \multicolumn{3}{c|}{ Masa seca raíz total $(\mathrm{mg})$} & \multicolumn{3}{c}{ Masa seca raicilla (mg) } \\
& $60 \mathrm{~d}$ & $90 \mathrm{~d}$ & $120 \mathrm{~d}$ & $60 \mathrm{~d}$ & $90 \mathrm{~d}$ & $120 \mathrm{~d}$ \\
\hline Testigo & $0,2 \mathrm{~b}$ & $1,5 \mathrm{~b}$ & $1,8 \mathrm{c}$ & $0,1 \mathrm{~b}$ & $1,2 \mathrm{~b}$ & $1,5 \mathrm{~b}$ \\
Glomus $\mathrm{sp1}$ & $0,9 \mathrm{a}$ & $1,6 \mathrm{~b}$ & $1,8 \mathrm{c}$ & $0,7 \mathrm{a}$ & $1,3 \mathrm{~b}$ & $1,5 \mathrm{~b}$ \\
Glomus $\mathrm{sp2}$ & $1,0 \mathrm{a}$ & $2,1 \mathrm{a}$ & $2,8 \mathrm{ab}$ & $0,7 \mathrm{a}$ & $1,8 \mathrm{a}$ & $2,4 \mathrm{ab}$ \\
G. clarum & $1,0 \mathrm{a}$ & $2,4 \mathrm{a}$ & $3,5 \mathrm{a}$ & $0,8 \mathrm{a}$ & $2,1 \mathrm{a}$ & $3,1 \mathrm{a}$ \\
G. intraradices & $1,1 \mathrm{a}$ & $1,7 \mathrm{~b}$ & $2,1 \mathrm{bc}$ & $0,8 \mathrm{a}$ & $1,3 \mathrm{~b}$ & $1,8 \mathrm{~b}$ \\
\hline
\end{tabular}

Letras diferentes representan diferencias significativas entre los tratamientos para $\mathrm{p}<0,05$.

Fitomasa seca total: los resultados (Cuadro 6) mostraron que a los 60 días el valor del testigo fue muy inferior (aproximadamente 4 veces) a los tratamientos con micorrizas. A los 90 días, los valores se diferencian en 2 grupos, el de mayor biomasa que corresponde a Glomus sp. 2, G. clarum y G. intraradices y el de menor biomasa seca el cual se corresponde con el testigo y Glomus sp. 1, que aunque muy cercano es superior. En el último período se mantiene la misma tendencia. En todos los períodos las plantas micorrizadas presentan los mayores valores de la fitomasa seca total con respecto al testigo.

Cuadro 6. Efecto de la inoculación, con 4 especies de HMA, en los tallos y las hojas (fitomasa seca aérea) y fitomasa seca total en plantas de tomate (Solanum lycopersicum L. Var., amalia) a los 60, 90 y 120 días de cultivo.

\begin{tabular}{lccc|ccc}
\hline \multicolumn{1}{c}{ Tratamientos } & \multicolumn{3}{c|}{ Fitomasa seca aérea (mg) } & \multicolumn{3}{c}{ Fitomasa seca total (mg) } \\
& $60 \mathrm{~d}$ & $90 \mathrm{~d}$ & $120 \mathrm{~d}$ & $60 \mathrm{~d}$ & $90 \mathrm{~d}$ & $120 \mathrm{~d}$ \\
\hline Testigo & $1400 \mathrm{~b}$ & $10500 \mathrm{c}$ & $12300 \mathrm{c}$ & $1600 \mathrm{~b}$ & $12000 \mathrm{c}$ & $14100 \mathrm{~b}$ \\
Glomus sp. 1 & $5200 \mathrm{a}$ & $11200 \mathrm{bc}$ & $13300 \mathrm{bc}$ & $6100 \mathrm{a}$ & $12800 \mathrm{bc}$ & $15100 \mathrm{~b}$ \\
Glomus sp. 2 & $5800 \mathrm{a}$ & $12500 \mathrm{ab}$ & $17300 \mathrm{a}$ & $6800 \mathrm{a}$ & $14700 \mathrm{ab}$ & $20100 \mathrm{a}$ \\
G. clarum & $5000 \mathrm{a}$ & $13100 \mathrm{ab}$ & $17700 \mathrm{a}$ & $6000 \mathrm{a}$ & $15500 \mathrm{a}$ & $21200 \mathrm{a}$ \\
G. intraradices & $6200 \mathrm{a}$ & $13700 \mathrm{a}$ & $15500 \mathrm{ab}$ & $7300 \mathrm{a}$ & $15300 \mathrm{a}$ & $17600 \mathrm{ab}$ \\
\hline
\end{tabular}

Letras diferentes representan diferencias significativas entre los tratamientos para $\mathrm{p}<0,05$.

Tasa de crecimiento relativo: a los 60 días se evidenciaron diferencias significativas (Cuadro 7) de las plantas micorrizadas en relación con el testigo que fue el menor. A los 90 días aunque se mantienen las diferencias significativas, ocurre en forma inversa y a los 120 días no hay diferencias significativas entre tratamientos.
Flores: en todos los períodos se observan diferencias significativas en relación con el testigo (Cuadro 8), a los 60 días el valor del testigo es aproximadamente 6 veces menor con respecto a G. intraradices que presentó la mayor cantidad de flores con 19. A los 90 días el testigo y Glomus sp. 2 tienen la mayor cantidad de flores, mientras 
Cuadro 7. Tasa de crecimiento relativo con 4 especies de HMA, en plantas de tomate (Solanum lycopersicum L. Var., amalia) a los 60, 90 y 120 días de cultivo.

\begin{tabular}{|c|c|c|c|}
\hline \multirow[t]{2}{*}{ Tratamientos } & \multicolumn{3}{|c|}{$\begin{array}{l}\text { Tasa de crecimiento relativo } \\
\qquad\left(\mathrm{mg} \cdot \mathrm{g}^{-1} \cdot \mathrm{dí}^{-1}\right)\end{array}$} \\
\hline & $60 d$ & $90 \mathrm{~d}$ & $120 d$ \\
\hline Testigo & $65 \mathrm{~b}$ & $83 \mathrm{a}$ & 5 \\
\hline Glomus sp 1 & $114 \mathrm{a}$ & $27 \mathrm{~b}$ & 5 \\
\hline Glomus sp 2 & $101 \mathrm{a}$ & $27 \mathrm{~b}$ & 10 \\
\hline G. clarum & $116 \mathrm{a}$ & $32 \mathrm{~b}$ & 11 \\
\hline \multirow[t]{2}{*}{ G. intraradices } & $120 \mathrm{a}$ & $23 \mathrm{~b}$ & 7 \\
\hline & & & NS \\
\hline
\end{tabular}

Letras diferentes representan diferencias significativas entre los tratamientos para $p<0,05$.

NS (no existe diferencias significativas).

Cuadro 8. Efecto de la inoculación, con 4 especies de HMA, en el número de flores, número de tomates, masa fresca del fruto e índice de cosecha en plantas de tomate (Solanum lycopersicum L. Var., amalia) a los 60,90 y 120 días de cultivo.

\begin{tabular}{lccc|cc|cc|cc}
\hline \multirow{2}{*}{ Tratamientos } & \multicolumn{3}{c|}{ Número de flores } & \multicolumn{2}{|c|}{ Número tomates } & \multicolumn{2}{|c|}{ Masa fresca fruto (g) } & \multicolumn{2}{|c}{ Índice de cosecha (\%) } \\
& $60 \mathrm{~d}$ & $90 \mathrm{~d}$ & $120 \mathrm{~d}$ & $90 \mathrm{~d}$ & $120 \mathrm{~d}$ & $90 \mathrm{~d}$ & $120 \mathrm{~d}$ & $90 \mathrm{~d}$ & $120 \mathrm{~d}$ \\
\hline Testigo & $2,8 \mathrm{c}$ & $14,8 \mathrm{a}$ & $3,8 \mathrm{c}$ & $6,2 \mathrm{~b}$ & $5,4 \mathrm{~b}$ & $44,1 \mathrm{~b}$ & $111,0 \mathrm{ab}$ & $35,1 \mathrm{~b}$ & $54,7 \mathrm{ab}$ \\
Glomus sp 1 & $14,6 \mathrm{~b}$ & $1,4 \mathrm{c}$ & $4,2 \mathrm{c}$ & $11,0 \mathrm{a}$ & $6,0 \mathrm{ab}$ & $74,4 \mathrm{a}$ & $166,7 \mathrm{a}$ & $48,5 \mathrm{a}$ & $65,0 \mathrm{a}$ \\
Glomus sp 2 & $13,0 \mathrm{~b}$ & $14,0 \mathrm{a}$ & $15,4 \mathrm{ab}$ & $7,8 \mathrm{~b}$ & $9,2 \mathrm{a}$ & $62,9 \mathrm{~b}$ & $106,8 \mathrm{~b}$ & $40,4 \mathrm{~b}$ & $46,1 \mathrm{~b}$ \\
G. clarum & $12,6 \mathrm{~b}$ & $9,4 \mathrm{~b}$ & $18,6 \mathrm{a}$ & $7,8 \mathrm{~b}$ & $5,4 \mathrm{~b}$ & $60,8 \mathrm{~b}$ & $89,7 \mathrm{~b}$ & $38,5 \mathrm{~b}$ & $42,1 \mathrm{~b}$ \\
G. intraradices & $19,0 \mathrm{a}$ & $6,4 \mathrm{~b}$ & $9,4 \mathrm{bc}$ & $6,8 \mathrm{~b}$ & $6,8 \mathrm{ab}$ & $58,7 \mathrm{~b}$ & $131,8 \mathrm{ab}$ & $40,3 \mathrm{~b}$ & $56,3 \mathrm{ab}$ \\
\hline
\end{tabular}

Letras diferentes representan diferencias significativas entre los tratamientos para $\mathrm{p}<0,05$.

Glomus sp. 1 y $G$. intraradices presentan el menor número de flores. A los 120 días, el testigo y Glomus sp. 1 muestran cifras inferiores de flores, sobresalen los tratamientos con G. clarum y Glomus sp. 2 con los máximos valores e intermedio se encuentra el $G$. intraradices.

Frutos y masa fresca de tomates: a los 90 días el tratamiento con Glomus sp. 1 produjo casi el doble de frutos que el testigo (Cuadro 8) y mayor cantidad que los otros tratamientos micorrizados con diferencias significativas. A los 120 días la cantidad de frutos presenta diferencias pequeñas entre los tratamientos y el testigo, excepto el tratamiento con la cepa Glomus sp. 2 que presentó 1,7 veces más frutos.

Los valores de la masa fresca del fruto (Cuadro 8) muestran que a los 90 días los tratamientos micorrízicos presentan pesos superiores al testigo, específicamente con la inoculación con Glomus sp. 1 que tuvo los mayores valores. A los 120 días el comportamiento es diferente, ya que el testigo produjo frutos con pesos que no difieren significativamente del resto de los tratamientos. Glomus sp. 1 presenta los valores más altos en los 2 tiempos analizados. 
Índice de cosecha: el resultado indicó que el tratamiento con la cepa Glomus sp. 1 alcanzó los valores más altos en los 2 períodos analizados, con diferencia significativa a los 90 días con todos los tratamientos (Cuadro 8). A los 120 días los tratamientos con las cepas Glomus sp. 2 y G. clarum mostraron menor índice de cosecha e inferior valor al testigo.

\section{DISCUSIÓN}

La evaluación de las variables en estos períodos corresponden a cambios que ocurren durante el desarrollo de las plantas de tomates; estos cambios interactúan de forma conjunta e incidieron positiva o negativamente en las diferentes variables que a su vez pudieron diferir en su comportamiento, debido a la cepa micorrízica que se encuentre asociada a la planta.

\section{Variables micorrízicas}

Los resultados con Glomus sp. 1, Glomus sp. 2 y G. clarum presentan una dinámica de colonización micorrízica similar entre ellos y diferente de G. intraradices. Las 3 primeras cepas sugieren que este comportamiento es el más generalizado para este género, lo que se confirma con los resultados de Dell' Amico et ál. (2007) quienes reportaron valores similares en el mismo cultivo con el empleo de una cepa de Glomus (INCAM 4) en diferentes dosis y 2 soportes diferentes, los valores que obtuvieron estos autores están entre 64 y $83 \%$ en los diferentes tratamientos y Gómez et ál. (2008) con valores de colonización entre 60 y $78 \%$ en 4 tratamientos.

La cepa de G. clarum presentó a los 60 días un valor de $84 \%$, superior a los resultados de Terry y Leyva (2006), que reportaron valores de $47 \%$ en el cultivo de tomate en campo por medio de una cepa de Glomus clarum de otro origen, esta variabilidad puede estar dada por adaptación a factores ambientales, condiciones del experimento y a que son diferentes aislados geográficos (Smith y Read 2008).

Ley et ál. (2009) obtuvieron valores de 45 a $86 \%$ en Sorghum bicolor con G. intraradices
(México), G. mosseae y Glomus sp., en 2 sustratos diferentes, los cuales son inferiores al rango obtenido en el experimento que comprende valores de 63 a 95\%, pero observándose que los valores más elevados son del G. intraradices al igual que el presente estudio.

Los resultados de DV obtenidos coinciden con los reportados por Fernández et ál. (2006) quienes obtuvieron valores hasta $3,2 \%$ en el cultivo de tomate con una cepa de Glomus (INCAM 4) y son superiores a las obtenidas por Mujica et ál. (2014) con valores entre 0,9 y $1,4 \%$ a partir de G. cubense en diferentes concentraciones.

El desarrollo del ED se comportó de forma diferenciada entre los 4 tratamientos, lo cual permite suponer diferentes estrategias para el intercambio de nutrientes entre ambos simbiontes que mostraron que algunas especies y morfoespecies benefician más a un hospedero que a otro y pueden adaptarse mejor a determinadas condiciones edafoclimáticas lo cual coincide con lo planteado por Linderman y Davis (2004) en que las relaciones HMA-plantas no son siempre iguales.

Gómez et ál. (2008) obtuvieron valores (alrededor de $12 \mathrm{mg}^{-1} \mathrm{~g}^{-1}$ ) comprendidos en el rango nuestro, que son muy cercanos a los valores de G. clarum y Glomus sp. 2. Se observa como la cepa Glomus sp. 1 presenta los valores más bajos en toda su dinámica, pero se muestra más eficiente ya que es capaz de mantener un buen nivel de intercambio de nutrimentos y fotosintatos superior a las otras cepas, más próximo al rango superior entre $4-20 \%$ que plantea Douds et ál. (2000), que se manifiesta en la producción de frutos de tomate con un índice de cosecha superior.

De forma contraria, $G$. intraradices presentó los valores más elevados de endófito al presentar mayor biomasa asociada a la raíz, lo que implica una demanda mayor de fotosintatos y tasa metabólica según Sánchez de Prager (2007); si esta compensación no se estableciera, el crecimiento del hospedero se vería seriamente afectado.

Los resultados de la CM, DV y ED sugiere que puede considerarse a $G$. intraradices como una cepa con alto poder de infectividad, 
o bien, un hongo que coloniza rápidamente y presenta una elevada ocupación fúngica en las raicillas de las plantas.

La cepa $G$. intraradices produce elevada cantidad de estructura fúngica, esto hace que la cantidad de nutrientes que necesita la planta para satisfacer las demandas de ambos simbiontes, sea alta y una forma de ser más eficiente funcionalmente es por medio de la absorción de más nutrientes mediante las hifas del micelio externo (Sánchez de Prager 2007), sin embargo, la capacidad de producir cantidad de micelio externo y su efectividad en absorber nutrientes depende de la cepa (Smith y Read 2008).

Los mayores valores se registraron a los 90 días, con excepción de la cepa Glomus sp. 2 que lo presentó a los 120 días. Esto puede deberse a que a los 90 días la actividad de floración y fructificación coinciden en su desarrollo y las plantas tienen como objetivo principal el crecimiento de los frutos lo que de forma general demandan más nutrientes y el aumento del micelio externo permite explorar mayor volumen y acceder a micrositios más finos del suelo $(<9 \mu \mathrm{m})$ a los que no pueden llegar las raicillas por presentar un diámetro mayor (Sánchez de Prager 2007).

La cantidad de esporas sugiere el potencial de inoculación que puede quedar en el suelo después de la cosecha y esto depende de varios factores como el tipo de cepa, su interacción con el hospedero, etc. (Smith y Read 2008). Las 3 primeras especies se comportan diferente al $G$. intraradices el cual muestra la capacidad de producir elevadas cantidades de esporas, aspecto que la caracteriza, fundamentado por los resultados de varios trabajos por medio de diferentes tipos de sustratos y hospederos (Ley et ál.2009, Mujica 2009, Navarro 2009).

Los resultados con rangos ligeramente mayores muestran similitud con los de Thougnon et ál. (2014) quienes obtuvieron producciones para utilizar como inóculo entre 800 y 11750 esporas. $\mathrm{kg}^{-1}$ y son inferiores a los de Vázquez et ál. (2010) que reflejan producciones de 5000, 12 000 y 18000 en cada período analizado. Aunque el objetivo de la investigación fue la producción de frutos, se identificó que la inoculación con $G$. intraradices también deja en el suelo una cantidad de esporas como potencial de inoculación para el siguiente cultivo.

\section{Variables de crecimiento}

A los 60 días se observó una mejor calidad y madurez en las plantas micorrizadas sobre el testigo. Los 2 momentos siguientes donde comienza la formación y crecimiento de frutos, pasaron a ser el objetivo principal del proceso de crecimiento, al provocar una evolución menos rápida en las otras variables. Las ventajas y beneficios que aportan las micorrizas fueron también evidenciadas por otros autores en diferentes ecosistemas agrícolas y forestales (Azcón y Barea 1992, Gianinazzi et ál. 2010).

En cuanto a la altura resultados similares obtuvo Ávila (2005) con la aplicación de diferentes dosis de inoculante micorrizógeno quien observó diferencias significativas con el testigo y Rodríguez (2009) al aplicar micorrizas sola y combinada, cuyos resultados siempre fueron superiores al testigo. Esta variable no mantiene el mismo comportamiento a través del tiempo cuando comenzó la producción de frutos pues estos son mayores en cantidad y tamaño en los tratamientos micorrizados, lo que implica menor producción de biomasa vegetal, a diferencia del testigo que mostró un crecimiento más rápido que acortó las diferencias con los otros tratamientos.

El incremento del número de hojas en los tratamientos micorrizados fue correspondido con los resultados de Rodríguez (2009) y Lagos (2010) al obtener un diámetro de copa mayor y más hojas en plantas micorrizadas respectivamente. Este efecto es debido a que las plantas micorrizadas necesitan mayor cantidad de fotosintatos para satisfacer su demanda y la del micosimbionte de tal forma que permita sin afectaciones el crecimiento y desarrollo estable de ambos organismos (Sánchez de Prager 2007). En cuanto al testigo, no es de esperar este rápido incremento del área foliar pues la planta solo debe satisfacer sus propias demandas. 
A partir de los 90 días comenzaron a diferenciarse las cepas Glomus sp. 2 y G. clarum como productoras de biomasa foliar y el testigo, Glomus sp. 1 y G. intraradices mantuvieron los niveles menores en la producción de hojas pero los 2 últimos superiores al testigo, lo que indicó una actividad fotosintética más alta que se justifica por la presencia simbiótica del HMA.

El diámetro del tallo tuvo un desarrollo estable con las diferencias en valores del testigo, mientras que los tratamientos micorrizados fueron mayores debido a las ventajas que proporciona la simbiosis micorrízica. Novella (2001) obtuvo similares resultados, no significativos estadísticamente pero los valores con HMA fueron mayores que los del testigo.

La disminución de la diferencia a los 90 días se explica porque el testigo en esta etapa se desarrolló vegetativamente más rápido que los tratamientos micorrizados que lo hicieron a los 60 días en etapa de floración y fructificación procesos que disminuyen la velocidad de engrosamiento del tallo; sin embargo, los valores de los tratamientos micorrizados siempre superan al testigo lo que implica un mejor desarrollo vegetativo, que continuó manifestándose hasta los 120 días.

Se observó una influencia positiva de las micorrizas en el desarrollo del sistema radicular de las plantas con variaciones según la especie de HMA; siempre mantuvieron diferencias con el testigo con excepción del Glomus sp. 1 el cual fue igual a los 120 días pero con un desarrollo mayor en número de hojas y grosor del tallo. Iguales resultados presentó Lagos (2010) al obtener tratamientos micorrizados con mayor fitomasa seca subterránea con respecto al no inoculado, pero en ocasiones fueron iguales los valores en ambos tratamientos pero el número de hojas y la altura de los tratamientos con micorrizas fueron mayores.

Los resultados de la fitomasa seca aérea y fitomasa seca total se debieron a que las plantas micorrizadas presentaron una mejor nutrición por la mayor absorción de nutrientes y agua (Cuenca et ál. 2003, Azcón et ál. 2009). Con diferentes cantidades de inóculo de HMA se observaron resultados iguales en el comportamiento de la fitomasa seca aérea que son mayores que la del testigo (Mujica 2014). Los valores siempre superiores al testigo de los tratamientos micorrizados y diferentes entre ellos confirmaron una relación beneficiosa de estos HMA con el cultivo de tomate y a su vez un comportamiento diferencial de las cepas en asociación con este hospedero en las condiciones experimentales en que fueron desarrolladas.

La TCRs nos expresa que a los 60 días las plantas micorrizadas alcanzaron un avanzado desarrollo vegetativo a diferencia del testigo, sin embargo, a los 90 días, aunque se mantuvieron las diferencias significativas, ocurrió en forma inversa, debido a que las plantas micorrizadas que llegaron a su madurez estaban en la fase de floración y fructificación, con su energía dedicada a estos procesos, mientras que el testigo aun no tenía su máximo desarrollo.

Se destaca que el máximo valor de la TCRs alcanzado por el testigo $\left(83 \mathrm{mg} \cdot \mathrm{g}^{-1} \cdot \mathrm{día}^{-1}\right)$ nunca supera el valor mínimo de los tratamientos micorrizados (101 mg.g ${ }^{-1} \cdot$ día $\left.^{-1}\right)$ en el primer período. A los 120 días no existieron diferencias pues ya todas las plantas estaban fructificadas y en fase final de su ciclo de vida.

La TCRs corrobora que al aplicar inóculos micorrízicos se pueden obtener plantas que se desarrollen en menor tiempo, con una ganancia de más de $100 \mathrm{mg} \cdot \mathrm{g}^{-1}$.día $\mathrm{a}^{-1}$ de biomasa seca total superior a la del testigo que solo alcanzó, en el primer período, $65 \mathrm{mg} \cdot \mathrm{g}^{-1}$.día ${ }^{-1}$.

En el estudio no se cuantificó la cantidad de fósforo $(\mathrm{P})$ en la planta, pero se conoce que dentro de sus múltiples funciones tiene un efecto elevado en el desarrollo de las flores y su deficiencia provoca la caída de las mismas, retarda la diferenciación de las yemas florales que conlleva a una disminución de frutos por plantas (Hernández 2000, Ávila 2005). La marcada diferencia en el número de flores entre los tratamientos micorrizados y el testigo a los 60 días fue debido 
a la cantidad de $\mathrm{P}$ absorbido por las plantas, que fue mayor en los tratamientos micorrizados, fundamentado por diferentes autores en los que se corrobora que las micorrizas toman de forma mas eficiente los fosfatos en el suelo a través de sus hifas que incrementan el volumen de suelo explorado (Sánchez de Prager 2007, Smith et ál. 2011).

Después de los 90 días, el comportamiento del testigo aunque con diferencias en el tiempo, elevó la cantidad de flores; este efecto pudo deberse al desarrollo tardío de la planta de testigo con respecto a los tratamientos micorrizados y a la utilización de macetas que limita la cantidad de sustrato y la disponibilidad de nutrientes.

El tratamiento con la cepa Glomus sp. 1 produjo 11 frutos como promedio por planta; dicho aspecto pudiera mejorar cuando se aplique en campo en iguales condiciones de suelo pues no estarían presentes las limitantes que conllevan los experimentos en macetas con respecto a los nutrientes. Comparados con los datos obtenidos por otros investigadores se califica como satisfactorio aunque es inferior a los 15 frutos producidos como promedio en campo por Mujica (2009) quien inoculó de forma independiente 3 especies de HMA (Glomus hoi-like, G. intraradices y Glomus mosseae) y Terry (2005) quien utilizó Glomus clarum con aplicación de 120 kg.ha- ${ }^{-1}$ de N. Se destaca que no se aplicó fertilización o enmienda orgánica, lo que conlleva a la disminución de nutrientes con el tiempo.

Los valores de la masa fresca del fruto estaban ligados al IC; este último mostró la producción de biomasa aérea que la planta desvió para la formación de frutos, donde la cepa Glomus sp. 1 derivó fotosintatos hacia la formación y crecimiento de los frutos seguido de $G$. intraradices.

El comportamiento de las cepas Glomus sp. 2 y G. clarum sugiere que retarda la producción de frutos, lo que hace poco efectiva su producción. En el caso de Glomus sp. 2 presentó a los 120 días la mayor cantidad promedio de frutos $(9,2)$. El IC obtenido con la inoculación de esta especie fue uno de los menores en todos los tratamientos ensayados, ya que los frutos no crecieron al mismo ritmo que en el resto de los otros tratamientos y esto pudo deberse a que esta cepa dirigió la mayor parte de su energía a la formación de biomasa total sin considerar el fruto que alcanza 20,1 g mientras que los valores de los otros tratamientos varían de 14,1 a 17,6 g.

Otra causa que incidió en todo el proceso de la formación final del fruto fue la temperatura superior a $\operatorname{los} 30^{\circ} \mathrm{C}$, que se presentó en 49 días durante el cultivo a partir de los 60 días de crecimiento de las plantas. Los valores de temperaturas se manifestaron en períodos entre 3 y 9 horas al día, los cuales incidieron en el desarrollo de las flores y su aborto, con un resultado final de una disminución en el rendimiento. Dichos aspectos coinciden con los obtenidos por Mutton et ál. (1987) y de Konning (1989) en cuanto a las consecuencias de la incidencia de temperaturas superiores a $30^{\circ} \mathrm{C}$ en el cultivo de tomate, refiriéndose además a la disminución de la fertilidad del grano de polen y al cuajado de los frutos.

\section{CONCLUSIONES}

Se diferencia la efectividad de las cepas Glomus sp. 1, Glomus sp. 2, G. clarum y G. intraradices en la productividad del cultivo de tomate.

Las cepas Glomus sp. 1 y G. intraradices mostraron mayor eficiencia en el incremento de la biomasa de los frutos.

Las variables de crecimiento y micorrízicas varían su comportamiento según el estadío en que se encuentra la planta.

Los HMA se benefician en forma diferente según la planta hospedera y ellas responden diferencialmente según la cepa que se utilice.

\section{AGRADECIMIENTOS}

A la Dra. Sara Herrera Figueroa por sus consejos y observaciones y al Técnico Osbel Gómez Ricardo por su labor y apoyo en el desarrollo del experimento. 


\section{LITERATURA CITADA}

ÁVILA O.L. 2005. Análisis de diferentes dosis de micorrizas (EcoMic) en producción de posturas de tomate (Lycopersicon esculentum Mill.) y su influencia en el rendimiento del cultivo. Tesis de maestría, Universidad Agraria de La Habana, Cuba. 71 p.

AZCÓN C., BAREA J.M. 1992. Interactions between mycorrhizal fungi and other rhizosphere microorganisms, pp. 163-198. In: M.F. A. Chapman and Hall (eds.) Micorrhizal Functioning. An Integrative Plant-Fungal Process. New York.

AZCÓN C., BAREA J.M., GIANINAZZI S., GIANINAZZI, V. 2009. Mycorrhizas-Functional Processes and Ecological Impact. Springer-Verlag Berlin Heidelberg. 239 p.

BAAR J. 2008. From production to application of arbuscular mycorrhizal fungi in agricultural systems: requirements and needs, pp. 361-374. In: Mycorrhiza. Third Edition. Ed. Ajit Varma.

BEABLE C.L. 1987. Plant growth analysis. Cap. 2. In: J. Coombs, P.O. Hall, S.P. Long and J.M.O. Scurlock (eds). Techniques bioproductivity and photosynthesis. Pergamon Press Ltd. 298 pp.

BEVER J.D. 2002. Negative feedback within a mutualism: Host-specific growth of mycorrhizal fungi reduces plant benefit: Proceedings of the Royal Society of London 269:2595-2601.

CUENCA G., DE ANDRADE Z., LOVERA M., FAJARDO L., MENESES E., MÁRQUEZ M., MACHUCA R. 2003. Pre-selección de plantas nativas y producción de inóculos de hongos micorrízicos arbusculares (HMA) de relevancia en la rehabilitación de áreas degradadas de la gran sabana, Estado de Bolivar, Venezuela. Ecotrópicos 16(1):27-40.

DE KONING A.N.M. 1989. The effect of temperature on fruit growth and fruit load of tomato. Acta Horticulturae 248:329-336.

DELL' AMICO J.M., FERNÁNDEZ F., NICOLÁS E., LÓPEZ L.F., SÁNCHEZ M.J. 2007. Respuesta fisiológica del tomate a la aplicación de dos inoculantes a base de Glomus $\mathrm{sp}_{1}$ (INCAM 4) por dos vías de inoculación diferentes. Cultivos Tropicales 28(2):51-58.

DOUDS D.D., PFEFFER P.E., SHACHAR Y. 2000. Carbon partitioning, cost and metabolism of arbuscular mycorrhizae in arbuscular mycorrhizas: physiology and function, pp.107-130. In: Y. Kapulnick y D.D. Douds Jr., (eds.). Arbuscular Mycorrhizas: Molecular Biology and Physiology. Kluwer Academic Publishers, Dordrecht, The Netherlands.

FERNÁNDEZ F., DELL' AMICO J.M., RODRÍGUEZ P. 2006. Efectividad de algunos tipos de inoculantes micorrízicos a base de Glomus hoi "like" en el cultivo del tomate (Lycopersicon esculentum Mill. Var. Amalia). Cultivos Tropicales 27(3):25-30.
GIANINAZZI S., GOLLOTTE A., BINET M., VAN TUINEN D., REDECKER D., WIPF D. 2010. Agroecology: the key role of arbuscular micorrizas in ecosystem services Mycorrhiza 20:519-530.

GIOVANETTI M., MOSSE B. 1980. An evaluation of techniques for measuring vesicular-arbuscular mycorrhizal infection in roots. New Phytol. 84:489-500.

GÓMEZ L., RODRÍGUEZ M., DE LA NOVAL B., MIRANDA I., HERNÁNDEZ M.A. 2008. Interacción entre el ECOMIC y una población cubana de Meloidogyne incognita en tomate. Rev. Protección Veg. 23(2):90-98.

HAO Z., FAYOLLE L., VAN TUINEN D., GIANINAZZI V., GIANINAZZI S. 2009. Mycorrhiza reduce development of nematode vector og Grapevine fanleaf virus in soils and root systems, pp. 100-1001. In: E. Boudon-Padfieu (ed.). Extended abstract 16th meeting of ICVG, Dijon, France.

HERNÁNDEZ M.I. 2000. Las micorrizas arbusculares y las bacterias rizosféricas como complemento de la nutrición mineral del tomate (Licopersicon esculentum Mill.). Tesis de maestría, Instituto de Investigaciones Hortícolas "Liliana Dimitrova", MINAG, $82 \mathrm{p}$.

HERRERA R.A., FURRAZOLA E., FERRER R.L., FERNÁNDEZ R. TORRES Y. 2004. Functional strategies of root hairs and arbuscular mycorrhizae in an evergreen tropical forest, Sierra del Rosario, Cuba. Revista CENIC Ciencias Biológicas 35(2):113-123.

IRVING L.J., CAMERON D.D. 2009. You are what you eat: interactions between root parasitic plants and their hosts. Adv. Bot. Res. 50:87-138.

KOIDE R.T., KABIR Z. 2000. Extraradical hyphae of the mycorrhizal fungus Glomus intraradices can hydrolyse organic phosphate. New Phytol. 148:511-517.

LAGOS S. 2010. Evaluación de cuatro cepas de micorriza arbuscular en plantas de tomate en vivero, Zamorano, Honduras. Proyecto especial de graduación del programa de Ingeniería Agronómica, Escuela Agrícola Panamericana, Zamorano. Honduras. 18 p.

LEY J.F. 2012. Eficiencia de cepas del género Glomus en cultivos de lechuga y tomate en condiciones controladas. Tesis de maestría, Instituto de Ecología y Sistemática, Cuba. 86 p.

LEY J.F., FURRAZOLA E., COLLAZO E., MEDINA M. 2009. Efecto de la aplicación de bentonita sobre la colonización micorrízica y la esporulación de hongos micorrizógenos. Acta Botánica Cubana 206:34-37.

LINDERMAN R.G., DAVIS E.A. 2004. Varied response of marigold (Tagetes spp.) genotypes to inoculation with different arbuscular mycorrhizal fungi. Sci. Hortic. 99:67-78.

MARULANDA A., BAREA J.M. 2009. Stimulation of plant growth and drought tolerance by native microorganisms (AM fungi and bacteria) from 
dry environments: mechanisms related to bacterial effectiveness. J. Plant Growth Regul. 28:115-124.

MUJICA Y. 2009. Efectividad de la inoculación con HMA en la nutrición del tomate (Solanum lycopersicum L.) en suelo ferralítico rojo lixiviado. Tesis de maestría, Instituto Nacional de Ciencias Agrícolas, Cuba. 73 p.

MUJICA Y., MENA A., MEDINA A., ROSALES P. 2014. Respuesta de plantas de tomate (Solanum lycopersicum L.) a la biofertilización líquida con Glomus cúbense. Cultivos Tropicales 35(2):21-26 .

MUTTON L., PATTERSON B.D., NGUYEN V.O. 1987. Two stages of pollen development are particularly sensitive to low temperature. Tomato Genet. Coop. Rpt. 37:56-57.

NAVARRO A. 2009. Efecto del estrés hídrico y la asociación micorrícica en el crecimiento, el desarrollo y el metabolismo de las especies reactivas del oxígeno en plantas de frijol negro (Phaseolus vulgaris L.). Tesis de maestría, Instituto de Ecología y Sistemática y Universidad de La Habana, Cuba. 73 p.

NAVARRO A., FURRAZOLA E., LEY J., HAMEL C., RODÉS R. 2010a. The role of arbuscular mycorrhizal fungi in reactive oxygen species metabolism under drought conditions in black bean (Phaseolus vulgaris L.). Saskatoon. Sesión de Póster Geología/Biología. Conference of the Canadian Society of Soil Science $\&$ Canadian Society of Agronomy. Sp.

NAVARRO A., FURRAZOLA E., RODÉS R., TORRES Y., COLLAZO E., GOMEZ O., MASSIA C., HAMEL C. 2010b. Influence of drought and arbuscular mycorrhizal fungi inoculation on growth and development of black bean (Phaseolus vulgaris L.). Saskatoon. Sesión de Póster Geología/Biología. Conference of the Canadian Society of Soil Science \& Canadian Society of Agronomy. Sp.

NOVELLA R. 2001. Participación de las micorrizas arbusculares y la fertilización nitrogenada en el crecimiento y la nutrición del tomate (Lycopersicon esculentum Mill.) en un suelo Ferralsol desaturado. Tesis de maestría, Instituto Nacional de Ciencias Agrícolas, INCA, Cuba. 74 p.

OROZCO M.O. 2002. Respuesta de Seis Leguminosas Arbóreas a la Inoculación Micorrízica con Suelos de Diferente Potencial de Colonización. Tesis de maestría, Instituto de Ecología y Sistemática, Cuba. 70 p.

PHILLIPS J.M., HAYMAN D.S. 1970. Improved procedures for clearing roots and staining parasitic and vesiculararbuscular mycorrhizal fungi for rapid assessment of infection. Trans. BR. Mycol. Soc. 55:158-161.

PORRAS A., SORIANO M.L., PORRAS A., AZCON R. 2009. Arbuscular mycorrhizal fungi increased growth, nutrient uptake and tolerance to salinity in olive trees under nursery conditions. J. Plant Physiol. 166:1350-1359.

RODRÍGUEZ B. 2009. Respuesta del tomate (Solanum lycopersicum L.) a la aplicación combinada de hongos micorrízicos arbusculares, un estimulador del crecimiento y fertilizantes minerales. Tesis de maestría, INCA, Cuba. 67 p.

SÁNCHEZ DE PRAGER M. 2007. Las Endomicorrizas: Expresión bioedáfica de importancia en el trópico. Palmira. Universidad Nacional de Colombia. Facultad de Ciencias Agropecuarias. 352 p.

SMITH S.E., READ D.J. 2008. Mycorrhizal symbiosis, $3^{\mathrm{a}}$ ed. Academic, London. 815 p.

SMITH S.E., JAKOBSEN I., GRONLUND M., SMITH F.A. 2011. Roles of Arbuscular Mycorrhizas in Plant Phosphorus Nutrition: Interactions between Pathways of Phosphorus Uptake in Arbuscular Mycorrhizal Roots Have Important Implications for Understanding and Manipulating Plant Phosphorus Acquisition. Plant Physiology 156:1050-1057.

TERRY E. 2005. Microorganismos benéficos y productos Bioactivos como alternativas para la Producción ecológica de tomate (Lycopersicon esculentum, Mill. Var. "Amalia"). Tesis de doctorado, INCA, Cuba. 133 p.

TERRY E., LEYVA A. 2006. Evaluación agrobiológica de la coinoculación micorrizas-rizobacterias en tomate. Agronomía Costarricense 30(1):65-73.

THOUGNON A.J., EYHERABIDE M., ECHEVERRÍA H.E., SAINZ H.R., COVACEVICH F. 2014. Capacidad micotrófica y eficiencia de consorcios con hongos micorrícicos nativos de suelos de la provincia de Buenos Aires con manejo contrastante. Rev. Argent. Microbiol. 46(2):133-143.

VALLADARES F. 2004. Ecología del bosque mediterráneo en un mundo cambiante, pp. 191-227. Ministerio del Medio Ambiente. EGRAF, S.A., Madrid.

VÁZQUEZB.,RIVERA R.,FERNÁNDEZK., RODRÍGUEZ Y. 2010. Caracterización del comportamiento micorrízico en Brachiaria decumbens inoculada con Glomus hoi-like. cultrop. Consultado 30/01/15 Disponible en http://scieloprueba.sld. $\mathrm{cu} /$ scielo.php?script $=$ sci_arttext $\&$ pid $=\mathrm{S} 0258$ 59362010000300003\&lng=es\&nrm=iso

ILSON G.W.T., RICE C.W., RILLIG M.C., SPRINGER A., HARTNETT D.C. 2009. Soil aggregation and carbon sequestration are tightly correlated with the abundance of arbuscular mycorrhizal fungi: results from long-term field experiments. Ecol. Lett. 12:452-461.

Todos los derechos reservados. Universidad de Costa Rica. Este artículo se encuentra licenciado con Creative Commons Reconocimiento-NoComercial-SinObraDerivada 3.0 Costa Rica. Para mayor información escribir a rac.cia@ucr.ac.cr 
